\title{
Hall effect and transmission electron microscopy of epitaxial MnSi thin films
}

\author{
S. A. Meynell, ${ }^{1}$ M. N. Wilson, ${ }^{1}$ J. C. Loudon,${ }^{2, *}$ A. Spitzig, ${ }^{1}$ F. N. Rybakov, ${ }^{3}$ M. B. Johnson, ${ }^{1}$ and T. L. Monchesky ${ }^{1, \dagger}$ \\ ${ }^{1}$ Department of Physics and Atmospheric Science, \\ Dalhousie University, Halifax, Nova Scotia, Canada B3H $3 J 5$ \\ ${ }^{2}$ Department of Materials Science and Metallurgy University of Cambridge \\ 27 Charles Babbage Road Cambridge CB3 OFS, United Kingdom \\ ${ }^{3}$ Institute of Metal Physics, Ekaterinburg, 620990, Russia
}

(Dated: November 12, 2014)

\begin{abstract}
We present Hall effect measurements on $\mathrm{MnSi} / \mathrm{Si}(111)$ epilayers and find an anomalous Hall contribution that is significantly smaller than in bulk crystals, which enables the observation of an additional contribution to the anomalous signal previously overlooked in MnSi. Our measurements indicate the signal is not due to skyrmions in MnSi thin films, which are absent in out-of-plane fields, but rather are the result of scattering from the cone phase. The absence of magnetic contrast in the transmission electron microscopy (TEM) measurements are consistent with this interpretation. We provide a method to model TEM images of skyrmions lattices to determine the conditions necessary for their observation in other B20 epilayers with an anisotropy that is favourable to their formation.
\end{abstract}

PACS numbers: 73.50.Jt, 75.25.-j, 75.30.-m, 75.70.Ak

\section{INTRODUCTION}

Chiral interactions in magnetic systems are unique in their ability to stabilize 2-dimensional (2D) solitons known as skyrmions, as first predicted by Bogdanov et $a l .{ }^{1,2}$. The potential for these self-assembled magnetic nanostructures to be exploited in spintronic devices ${ }^{3-5}$ with high mobility ${ }^{6}$ currently makes them a topic of great interest. Investigations of the chiral B20 family of crystals have played a prominant role in the study of skyrmions. Skyrmions are observed in bulk B20 crystals in a small pocket of the phase diagram near the ordering temperature, referred to as the $A$-phase ${ }^{7-10}$. In contrast, nanostructures are found to provide important interactions by way of finite size effects, surface states ${ }^{11,12}$ and anisotropy ${ }^{13-15}$ that stabilize skyrmions over a broad temperature and field range. Examples include mechanically thinned crystals ${ }^{16-18}$, epitaxial films ${ }^{19-21}$, and nanowires ${ }^{22}$.

As conduction electron spins adiabatically follow the local magnetization of the skyrmions, they acquire a Berry phase that acts as an effective magnetic field, $B_{\text {eff }}{ }^{23,24}$. Since $B_{\text {eff }}$ is typically greater than $10 \mathrm{~T}^{25,26}$, this can lead to an appreciable topological Hall effect (THE). The appearance of a THE in the $A$-phase ${ }^{27}$ of bulk MnSi has led to the notion that a THE is proof of the existence of skyrmions. One of the difficulties with making such a conclusion is that there are many physical phenomena that give rise to Hall effects and the extraction a topological contribution amongst other contributions can be difficult. In this Paper, we argue that scattering from the cone phase produces a Hall resistance in $\mathrm{MnSi} / \mathrm{Si}(111)$ epilayers that has previously been misinterpreted as a THE from skyrmions.

Li et al. present Hall effect and Lorentz microscopy measurements of $\mathrm{MnSi}$ thin films grown on $\mathrm{Si}(111)$ substrates $^{28}$. From this data, they conclude that the ground state structure is helimagnetic with an in-plane

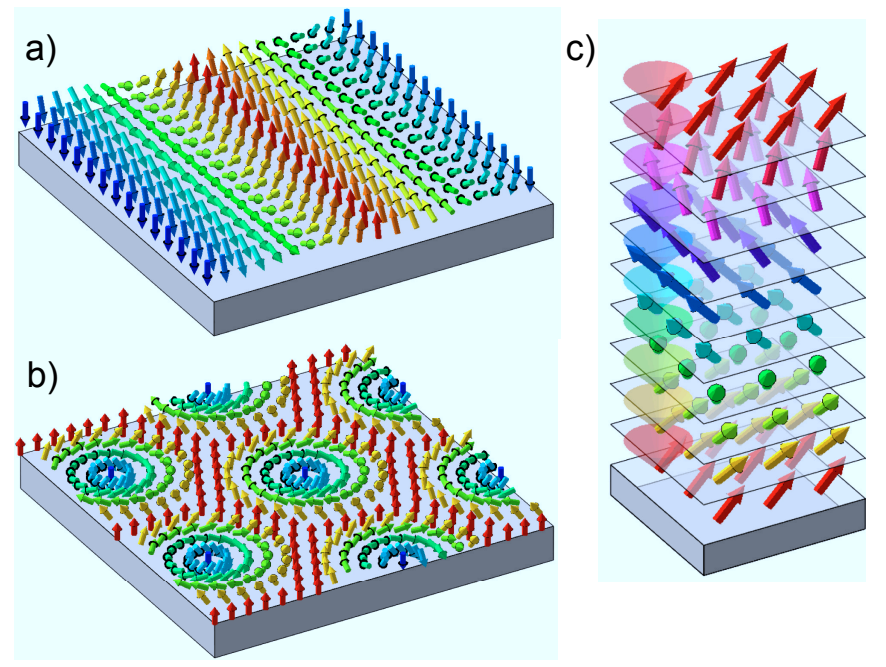

FIG. 1. (Color on-line) Some of the possible chiral magnetic states in B20 magnets in out-of-plane magnetic fields: (a) distorted helix (helicoidal phase), (b) skyrmion lattice phase, (c) cone phase.

propagation vector (Fig. 1(a)) and that skyrmions are produced in out-of-plane magnetic fields (Fig. 1(b)). These results are in contradiction with several papers on this material system ${ }^{29}$. Two complementary polarized neutron reflectometry measurements ${ }^{30,31}$ and SQUID magnetometry ${ }^{30}$ determine that the ground state helical structure propagates along the the out-of-plane [111] direction as a result of an easy-plane uniaxial anisotropy, the demagnetizing field and the exchange anisotropy ${ }^{32}$. In out-of-plane magnetic fields, there are no first-order magnetic phase transitions that would signal the appearance of skyrmions. This result is explained by theoretical analysis that shows the easy-plane anisotropy suppresses skyrmions in out-of-plane magnetic fields and makes the cone phase (Fig. 1(c)) the thermodynamically 
stable phase over the entire out-of-plane magnetic phase $\operatorname{diagram}^{15}$.

In order to address the discrepancy, we performed Hall effect measurements of $\mathrm{MnSi} / \mathrm{Si}(111)$ and simulated transmission electron micrographs of the skyrmions phase. We present Hall effect data in section III with an interpretation that is consistent with measurements of both thin films and bulk crystals. This has important implications not only for other B20 epilayers, including $\mathrm{FeGe} / \mathrm{Si}(111)^{20,33}, \mathrm{Fe}_{1-x} \mathrm{Co}_{x} \mathrm{Si} / \mathrm{Si}(111)^{25,34,35}$ and $\mathrm{Mn}_{1-x} \mathrm{Fe}_{x} \mathrm{Si} / \mathrm{Si}(111)^{36}$ and $\mathrm{MnGe} / \mathrm{Si}(111)^{37}$, but for bulk chiral magnets as well. In section IV we present calculations of the magnetic contrast produced in TEM images of skyrmions in thin films under optimal image conditions. These calculations provide a simple means of determining the material parameters necessary to observe skyrmions by TEM and at the same time reinforce our interpretation of the Hall effect data.

\section{SAMPLE PREPARATION}

MnSi thin films were grown with a thickness that ranged between $d=12.7-38.8 \mathrm{~nm}$. The films were grown by molecular beam epitaxy on insulating $\mathrm{Si}$ substrates (resistivity $\rho>50 \Omega \mathrm{m}$ ), as described in Ref. 30 , and were capped with a protective $20 \mathrm{~nm}$ thick amorphous Si layer. The four-probe resistivity measurements were done on pattern Hall bars with gold wire leads attached with indium solder. The samples were prepared by photolithographic patterning using SPR220 3.0 photoresist and were either wet-etched with a 45:35:15 acetic:nitric:hydrofluoric acid mixture, or were dry-etched with a $2 \mathrm{keV}$ Ar-ion gun. The residual resistivity ratios of the samples presented in Sect. III were 26.7 and 34.5. Plan-view and cross-sectional TEM specimens were prepared by low-angle mechanical polishing as described in Ref. 38 .

\section{HALL EFFECT}

\section{A. Standard Phenomenological Model}

The Hall effect in magnetic materials is typically broken into three terms. The diagonal component of the resistivity tensor,

$$
\rho_{y x}=\rho_{y x}^{O}+\rho_{y x}^{A}+\rho_{y x}^{T}
$$

includes contributions from the ordinary Hall effect $\rho_{y x}^{O}$, as well as the anomalous $\rho_{y x}^{A}$ and the topological $\rho_{y x}^{T}$ contributions. The ordinary contribution due to the Lorentz force $\rho_{y x}^{O}=\mu_{0} R_{0} H$ is expressed in terms of the Hall coefficient $R_{0}$ and applied magnetic field $\mu_{0} H$. The anomalous contribution,

$$
\rho_{y x}^{A}=\rho_{y x}^{\mathrm{ext}}+\rho_{y x}^{\mathrm{int}},
$$

is separated into extrinsic and intrinsic contributions and is usually assumed to be linear in the out-of-plane component of the magnetization $M$. The extrinsic term $\rho_{y x}^{\text {ext }}=$ $\alpha \rho_{x x} M+\beta \rho_{x x}^{2} M$ is composed of the skew scattering ${ }^{39}$, parametrized by $\alpha$, and side jump scattering ${ }^{40}$ described by the coefficient $\beta$. Until recently, elastic and inelastic scattering were considered to contribute equally to $\rho_{y x}$, which is not true in general ${ }^{41}$. The elastic component can be separated out by expressing the scattering in terms of the residual resistivity $\rho_{0}=\rho_{x x}(H=0, T=0)$. The skew scattering is then described by,

$$
\rho_{y x}^{\text {skew }}=\left(\alpha_{0} \rho_{0}+\alpha_{1}\left(\rho_{x x}-\rho_{0}\right)\right) M,
$$

which is parameterized in terms of the temperature independent coefficients $\alpha_{0}$ and $\alpha_{1}$. A similar decomposition of the side jump contribution $\rho_{y x}^{s j}$ is also necessary in general. Although the role of inelastic scattering remains an open question ${ }^{42}$, there is evidence to suggest that phonon contributions are small relative to defect scattering ${ }^{41,43,44}$. In the MnSi thin films, however, an inelastic term seems necessary as discussed below.

The original work of Karplus and Luttinger ${ }^{45}$ on the intrinsic origin of the Hall effect can be interpreted in terms of a Berry phase effect ${ }^{46}$. Due to the differences in topology of the Fermi surface for up and down spins, the Berry phase that the electrons acquire in momentumspace leads to an anomalous velocity responsible for an intrinsic contribution to the AHE. This contribution is given by $\rho_{y x}^{\text {int }} \sim \rho_{x x}^{2} M$ in Ref. 45 . The sensitivity of the intrinsic mechanism to the Fermi level is reflected in its temperature dependence ${ }^{47}$. Equations $(1)-(3)$ and $\rho_{y x}^{\text {int }}$ together with measurements of $\rho_{x x}(H, T)$ and $M(H, T)$ can be used to fit the $\rho_{y x}$ data to determine the corresponding coefficients. In the case of bulk MnSi crystals, Lee et al. ${ }^{48}$ are able to fit their data to $\rho_{y x}^{A}=S_{H} \rho_{x x}^{2} M$ with a temperature independent $S_{H}$-parameter. For their samples, which have a residual resistivity ratios in the range of 40 to 80 , they find that a skew scattering contribution is negligible, and argue that $\rho_{y x}^{A}$ is due only to intrinsic scattering.

In the case of epitaxial films where the residual resistivity ratios are lower, defect scattering cannot be ignored. $A b$ initio calculations recently demonstrated that sidejump and intrinsic contributions can be comparable in size ${ }^{49}$. Since, $\rho_{y x}^{s j}$ and $\rho_{y x}^{\text {int }}$ have the same functional form independent of scattering rate, it is very difficult to separate these contributions experimentally and therefore we group them into one term, $\rho_{y x}^{\text {int,sj }}=\rho_{y x}^{\text {int }}+\rho_{y x}^{s j}$,

$$
\rho_{y x}^{\text {int }, \mathrm{sj}}=b(T) \rho_{x x}^{2} M,
$$

and do not attempt to separate the elastic from the inelastic contribution to $\rho_{y x}^{s j}$.

A small THE is found in the $A$-phase of $\mathrm{MnSi}^{27}$, but was found to be an order of magnitude larger at high pressure $^{50}$. This discrepancy was found to be due to the difference in temperatures of the $A$-phases in $\mathrm{MnSi}$ under ambient and high pressure, as demonstrated by 
field cooling $\mathrm{MnSi}$ at ambient pressure through the precursor region ${ }^{26}$. This field cooling approach is able to catch metastable skyrmion lattices in a low temperature state ${ }^{15}$, as demonstrated in magnetic force microscopy measurements of $(\mathrm{Fe}, \mathrm{Co}) \mathrm{Si}^{51}$. The THE due to skyrmions,

$$
\rho_{y x}^{T} \simeq P R_{0} B_{\mathrm{eff}},
$$

depends on the polarization of the conduction electrons, $P$, and the effective magnetic field $B_{\text {eff }}=n_{s}(h / e)$ given by the skyrmion winding number density ${ }^{23}$ :

$$
n_{s}=\frac{1}{4 \pi} \mathbf{n} \cdot\left(\frac{\partial \mathbf{n}}{\partial x} \times \frac{\partial \mathbf{n}}{\partial y}\right),
$$

where $\mathbf{n}$ is the local direction of the magnetization. The integral of this quantity over the skyrmion unit cell gives the topological charge, which is -1 for skyrmions.

\section{B. Experiment}

Data were collected on a Physical Properties Measurement System (PPMS) system (made by Quantum Design) over a temperature range of $2-300 \mathrm{~K}$ in fields up to $9 \mathrm{~T}$. Because of the non-zero susceptibility above the saturation field, it is very difficult to separate $\rho_{y x}^{O}$ from $\rho_{y x}^{A}$. For bulk crystals, Lee et al. obtain a value of $R_{0}=7.32 \times 10^{-11} \Omega \mathrm{m} / \mathrm{T}$ from a fit to their data with $\rho_{y x}(H)=\rho_{y x}^{O}+S_{H} \rho_{x x}^{2} M$. Whereas the slope $\rho_{y x}(H)$ provides a second estimate of $R_{0}^{\prime}=17 \times 10^{-11} \Omega \mathrm{m} / \mathrm{T}$ at a temperature $T=2.8 \mathrm{~K}$ that is reported to be more consistent with the interpretation of the topological Hall effect $^{52}$.

In order to parametrize the high-field behavior of our samples, we fit $\rho_{y x}$ between $\mu_{0} H=7.5$ and $8.5 \mathrm{~T}$, far above the saturation field $\left(H_{C 2}\right)$, and assign the slope to the ordinary Hall coefficient $R_{0}^{\prime}$ and the intercept to the anomalous Hall contribution at high field, $\rho_{y x}^{A^{\prime}}$. We adopt a prime notation here to indicated parameters extracted from the behavior well above $H_{C 2}$. Figure 2 shows the temperature dependences of $R_{0}^{\prime}$ and $\rho_{y x}^{A^{\prime}}$. The ordinary contribution is comparable to the bulk value $R_{0}^{\prime}=17 \times$ $10^{-11} \Omega \mathrm{m} / \mathrm{T}^{52}$. However, the anomalous contribution dips near the Curie temperature $\left(T_{C} \simeq 42 \mathrm{~K}\right.$, Ref. 21$)$, with a value that is approximately $2-3$ times smaller in magnitude than the dip $\rho_{y x}^{A^{\prime}} \simeq-1.5 \times 10^{-9} \Omega \mathrm{m}$ found in bulk $\mathrm{MnSi}^{48}$.

We use the dependence of $\rho_{y x}^{A^{\prime}}$ on resistivity to gain some insight into the minimum phenomenological model necessary to fit the data for magnetic fields that are much higher than the fields where any contribution from a nonuniform magnetization might be present. In Fig. 3 we plot the quantity $\rho_{y x}^{A^{\prime}} / M$ versus $\rho_{x x}$, where the value for the magnetization at $\mu_{0} H=9 \mathrm{~T}$ is estimated from an extrapolation of the SQUID data between 3 and $5 \mathrm{~T}$ (see Fig. 10). If the Hall signal were predominantly due to defect and intrinsic/side-jump scattering, the data would

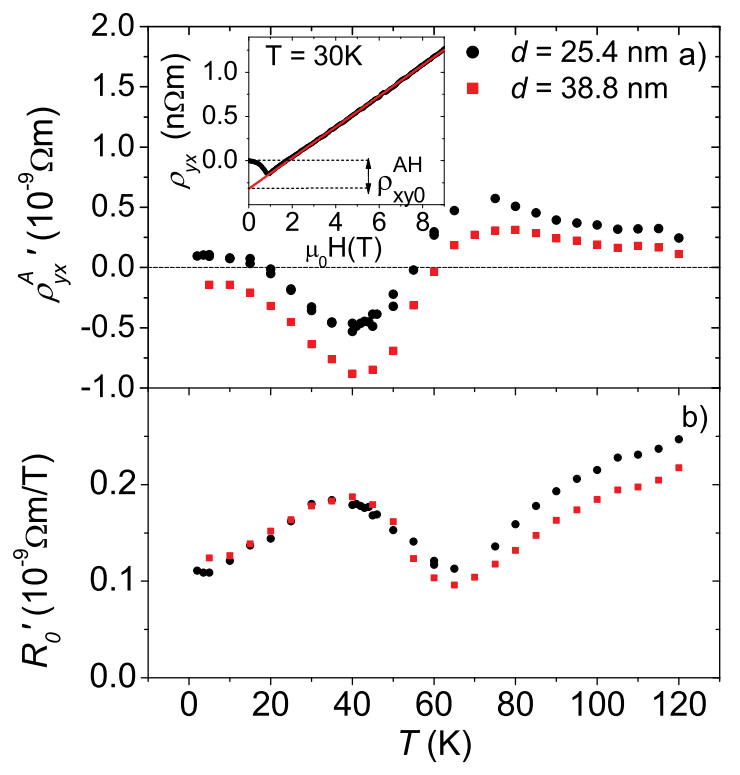

FIG. 2. (Color online) Temperature dependence of Hall effect for $d=25.4$ and $38.8 \mathrm{~nm}$ thick MnSi films. The inset shows the Hall resistivity for the $d=25.4 \mathrm{~nm}$ sample measured at $T=30 \mathrm{~K}$. The Hall resistivity is parameterized by the intercept (a), and slope (b) of the Hall resistance determined at fields above $\mu_{0} H=7.5 \mathrm{~T}$.

have parabolic distribution centred about $\rho_{x x}=0$ (assuming $b(T)$ is constant). The approximately linear dependence on $\rho_{x x}$ for lower $\rho_{x x}$ values requires the $\alpha_{1}$ term in Eq. (3) to fit the data. As shown in the analysis below, $b(T)$ is relatively small except at the lowest temperatures. The curvature in the data reflects either an intrinsic and/or a side jump contribution.

Complications arise at lower fields where $\rho_{y x}^{O}+\rho_{y x}^{\text {skew }}+$

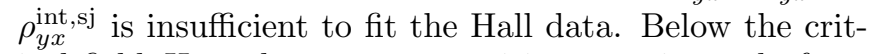
ical field $H_{C 2}$, the system transitions continuously from the field induced ferromagnetic state into a conical phase. The failure of this standard phenomenological model in the cone phase is reflected in a plot of $\left(\rho_{y x}-\rho_{y x}^{O}\right) / M$ versus $M^{2}$ in Fig. 4. The filled points correspond to fields below the saturation field, $H_{C 2}$. In this field range, the $\rho_{x x}$ does not vary appreciably (see Fig. 9) and therefore Fig. 4 should be approximately a straight line if the anomalous contribution followed the standard assumption, $\rho_{y x}^{A} \sim M$. The linear behavior of Fig. 4 reflects an $M^{3}$ dependence to the Hall effect below the saturation field. It is this dependence that produces the domeshaped feature in $\rho_{y x}^{A}$ at low field in Fig. 5. We find that the following form fits the data well:

$$
\rho_{y x}^{\text {other }}=c(T)\left(\frac{M}{M_{s}}-\left(\frac{M}{M_{s}}\right)^{3}\right),
$$

where $c(T)$ is a temperature dependent fitting parameter 


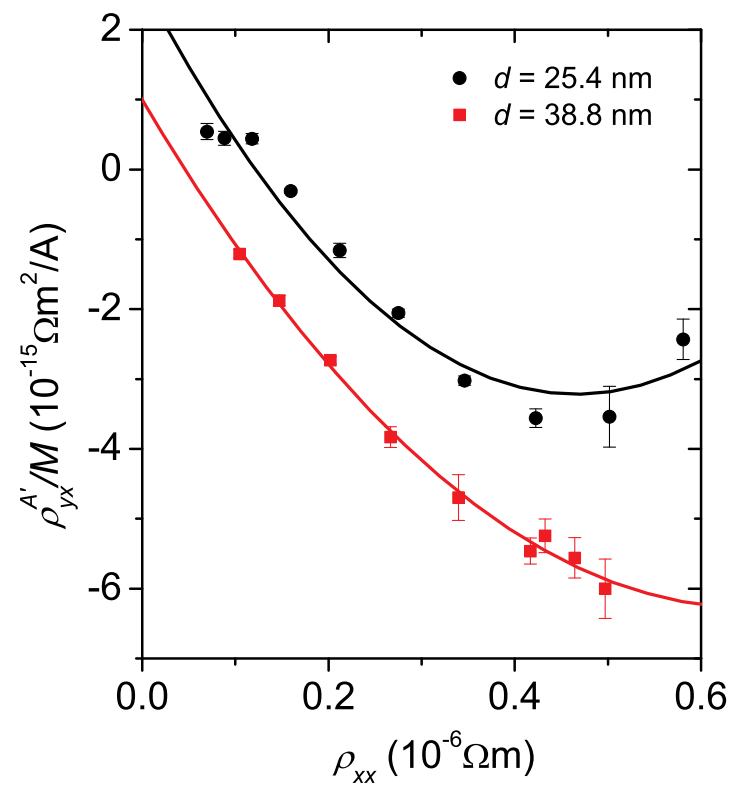

FIG. 3. The high-field anomalous Hall resistivity from Fig. 2(a) divided by the magnetization for the $25.4 \mathrm{~nm}$ and $38.8 \mathrm{~nm}$ samples between $T=5$ and $40 \mathrm{~K}$. The solid lines represent fits to the data with Eqs. (3) and (4). The values for $b(T)$ are given in Fig. 7(a).

and $M_{s}$ is the magnetization at the saturation field $H_{C 2}$. We set $\rho_{y x}^{\text {other }}=0$ for fields $H>H_{C 2}$ and therefore we do not consider the small increase in $M$ above $H_{C 2}$ in this term ${ }^{12}$, i.e. the contribution $\rho_{y x}^{\text {other }}$ is a term related to the magnetic texture below this field.

We fit $\rho_{y x}$ to Eqs. (1) - (4) and (7) with $R_{0}(T), \alpha_{0}$, $\alpha_{1}, b(T)$ and $c(T)$ as fitting parameters. The values for $R_{0}(T)$ and $\alpha_{0}$ are found from the high-field Hall measurements (Figs. 2(b) and 3). The parameter $\alpha_{0}$, which can vary substantially from sample to sample, as pointed out in Ref. 36, was found to be $10 \pm 3 \mathrm{~nm} / \mathrm{A}$ for the $25.4 \mathrm{~nm}$ sample and $-47 \pm 6 \mathrm{~nm} / \mathrm{A}$ for the $38.8 \mathrm{~nm}$ sample. The $c(T)$ parameter determined from the fit is approximately equal to the slope of Fig. 4 and is plotted in Fig. 7(c). The remaining two parameters, $\alpha_{1}$ and $b(T)$, were found iteratively by requiring that they fit both the high-field Hall data in Fig. 3 and the low field data if Fig. 5. The values for $b(T)$ are shown in Fig. $7(\mathrm{a})$ and we obtain $\alpha_{1}=28 \pm 2 \mathrm{~nm} /$ A for the $25.4 \mathrm{~nm}$ sample and $20 \pm 1 \mathrm{~nm} / \mathrm{A}$ for the $38.8 \mathrm{~nm}$ sample.

There is a clear difference in behavior between the value for $b$ in films and bulk. As $T$ increases above $15 \mathrm{~K}$, $b(T)$ falls by nearly an order of magnitude below the value of $S_{H}$ measured in bulk MnSi. This difference could be due to a combination of the difference in the intrinsic contribution that is very sensitive to changes in the Fermi surface that might be brought about by strain, and to

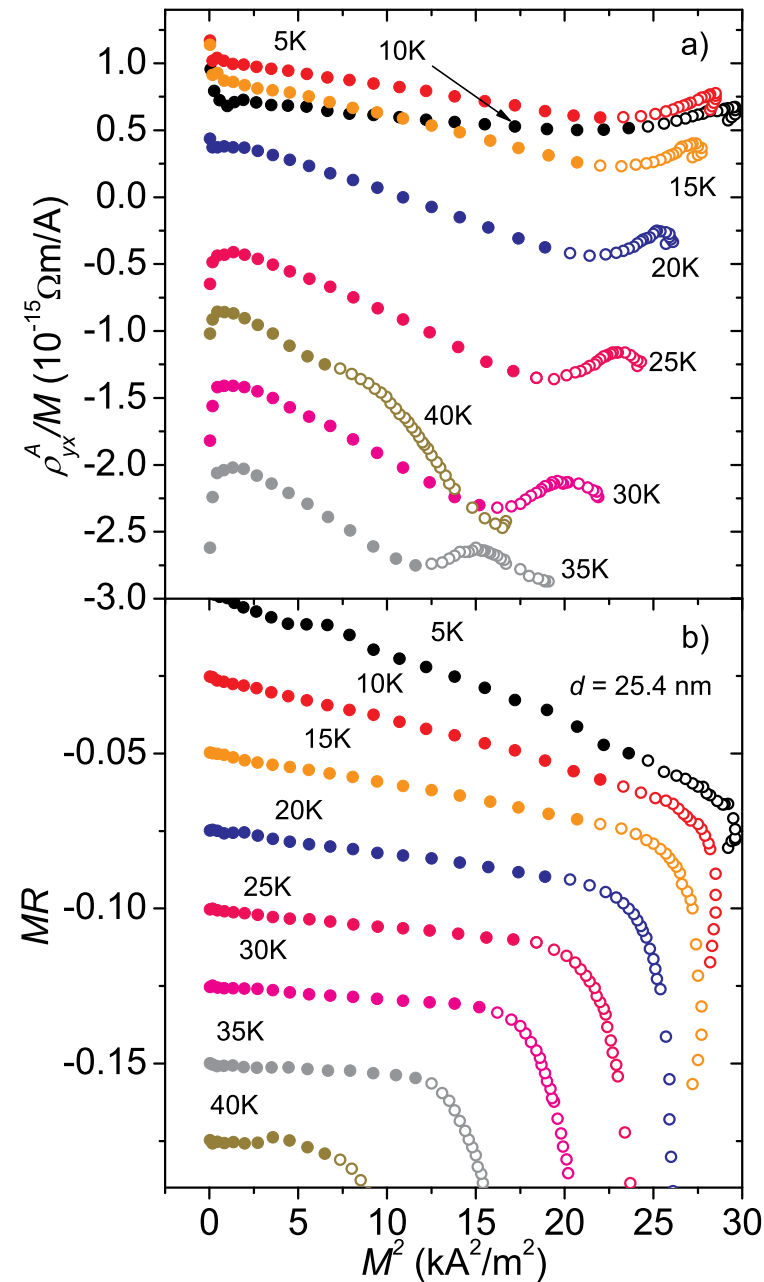

FIG. 4. (Color online) (a) Isothermal anomalous Hall resistivity $\rho_{y x}^{A}=\rho_{y x}-\rho_{y x}^{O}$ divided by the magnetization and (b) the magnetoresistance $\left(\rho_{x x}(H)-\rho_{x x}(0)\right) / \rho_{x x}(0)$ versus $M^{2}$ for a $d=25.4 \mathrm{~nm} \mathrm{MnSi} \mathrm{film} \mathrm{at} \mathrm{temperatures} \mathrm{between} T=5$ and $40 \mathrm{~K}$. The filled circles represent the data below the saturation field $H_{C 2}$ and the open circles represent the data above.

side jump scattering from the increase in the number of defects in films compared to bulk crystals.

Our fits to the Hall effect data interpret the small $\rho_{y x}^{A}$ observed in Fig. 2 as a competition between intrinsic scattering and extrinsic scattering. Alternatively, if the inelastic scattering parameter $\alpha_{1}$ is not included in the fit and the small $\rho_{y x}^{A}$ is interpreted in terms of an intrinsic/side-jump scattering term, the low-field fits are equally good. However, the high-field behavior fits much less well. Additionally, the $b$ parameter is much smaller in magnitude and opposite sign $\left(b \sim-0.007 \mathrm{~V}^{-1}\right)$ compared to bulk MnSi. Regardless of how we decompose the AHE data, the smaller AHE in films enabled us to uncover an additional contribution to the Hall effect in the 


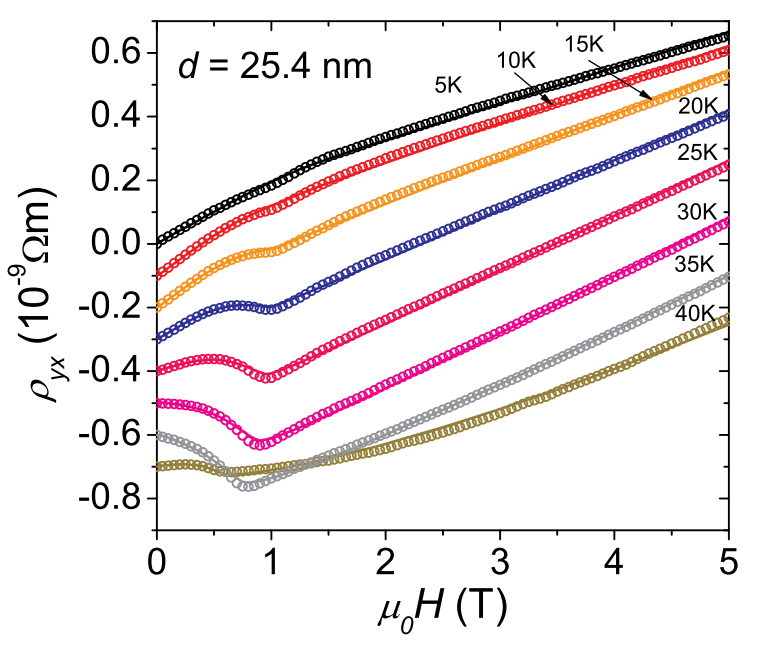

FIG. 5. (Color online) Isothermal Hall resistivity for the $d=$ $25.4 \mathrm{~nm} \mathrm{MnSi}$ film as a function of applied field. The lines represent simultaneous fits to the data below $\mu_{0} H=5 \mathrm{~T}$ and the high field data in Fig. 3. Data sets from neighbouring temperatures are offset vertically by $0.1 \times 10^{-9} \Omega \mathrm{m}$ from each other for clarity.

cone phase of $\mathrm{MnSi}$ that has previously been neglected as we describe below. Both fits yield the same $\rho_{y x}^{\text {other }}$ contribution to the Hall effect.

In Fig. 6(a), we use the fits to extract the $\rho_{y x}^{\text {other }}$ contribution from the data,

$$
\rho_{y x}^{\text {other }}=\rho_{y x}-\rho_{y x}^{O}-\rho_{y x}^{\text {skew }}-\rho_{y x}^{\text {int, sj }},
$$

which has the same size, sign and shape as the contribution attributed to a THE in Ref. 28. The sign of this contribution is positive, whereas Refs. 27 and 50 report a $\rho_{y x}^{T}<0$ in bulk crystlas. Li et al. attribute this difference to a possible reversal in the polarization of the conduction electrons in both their $10 \mathrm{~nm}$ and $50 \mathrm{~nm}$ thick MnSi films. Such a change in the density of states at the Fermi level seems unlikely given the small strain in a $50 \mathrm{~nm}$ thick film ${ }^{32}$. A second difficulty with attributing $\rho_{y x}^{\text {other }}$ to skyrmions is the absence of a discontinuity in $\rho_{y x}$ that would signal a first-order phase transition from a cone phase to a skyrmion phase, as is observed in bulk $\mathrm{MnSi}^{27,50}$. One might question whether disorder in the films' crystal structure might broaden such transitions, as observed in $\mathrm{Mn}_{1-x} \mathrm{Fe}_{x} \mathrm{Si}$ crystals ${ }^{26}$, and make them difficult to detect. However, the relatively sharp transitions into the skyrmion phase observed in $\mathrm{MnSi} / \mathrm{Si}(111)$ for in-plane magnetic fields would suggest otherwise ${ }^{21}$.

A comparison to measurements on bulk crystals suggests $\rho_{y x}^{\text {other }}$ is not due to a topological spin texture, but is due to a previously unaccounted intrinsic contribution. In Fig. 6(b) we plot $\rho_{y x}^{\text {other }}$ extracted from measurements on bulk crystals by Lee et al. ${ }^{48}$ for a measurement at $T=20 \mathrm{~K}$. Note that $\rho_{y x}^{\text {other }}$ for bulk MnSi has the same
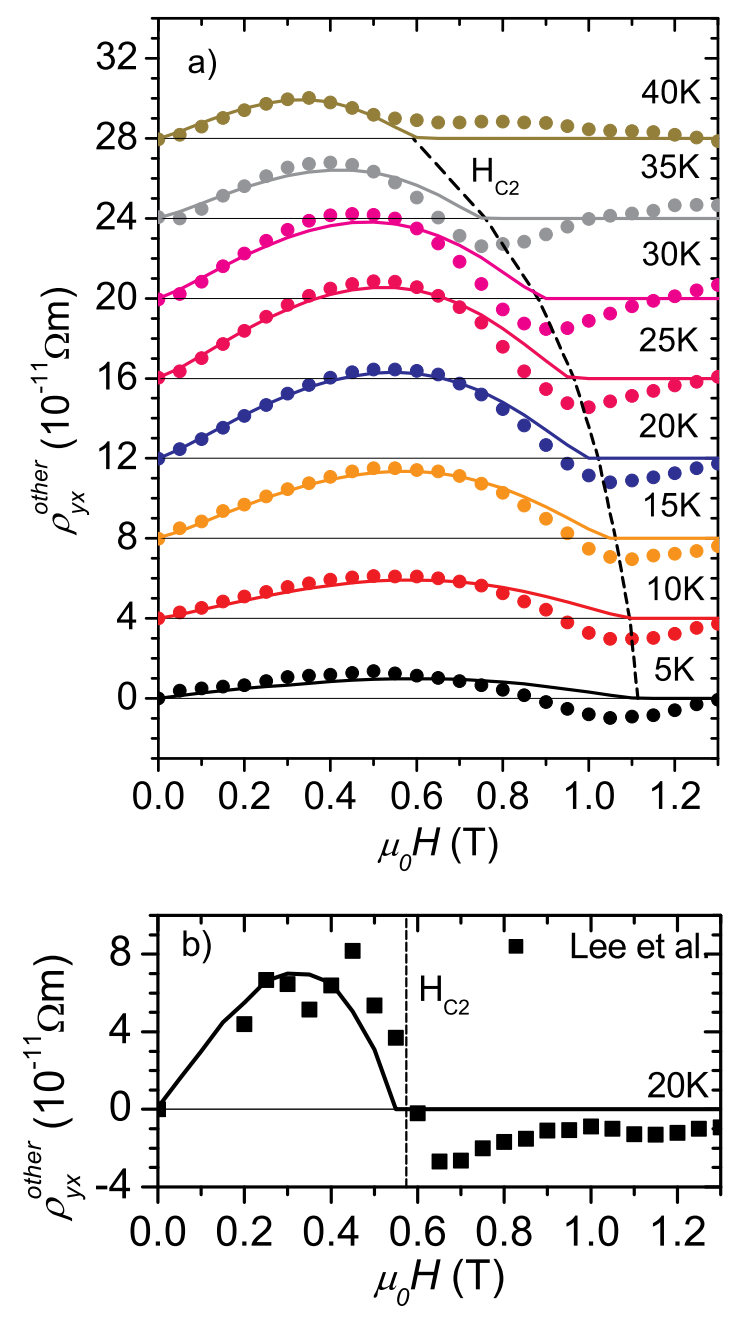

FIG. 6. (a) The difference between the measured and the fitted $\rho_{y x}$ (filled circles), as given by Eq. (8) at temperatures between $5 \mathrm{~K}$ and $40 \mathrm{~K}$. The solid line is a fit to this additional component to the Hall effect. The curves are offset vertically for clarity. (b) Evidence for the additional component in measurements in bulk MnSi crystals: the squares represent the difference between the measured and fitted Hall data from $T=20 \mathrm{~K}$ from Lee et al. ${ }^{48}$ The thick black is a fit to the difference using Eq. 7. The dashed lines in (a) and (b) indicate the saturation field $H_{C 2}$.

size and sign as $\rho_{y x}$ at $T=25 \mathrm{~K}$. The same $\rho_{y x}^{\text {other }}$ contribution is also present in the data in Ref. 53. In bulk samples at $T=20 \mathrm{~K}$, the cone phase is the thermodynamically stable phase below the field $H_{C 2}$, which does not contribute to $\rho_{y x}^{T}$ since it has zero topological charge. The reason that this term was perviously neglected was because $\rho_{y x}^{\text {other }} \approx 6 \times 10^{-11} \Omega \mathrm{m}$ is small compared to $\rho_{y x}^{A} \approx 40 \times 10^{-11} \Omega \mathrm{m}$ measured at $T=20 \mathrm{~K}$ in bulk MnSi.

The reason for the failure of the standard phenomeno- 


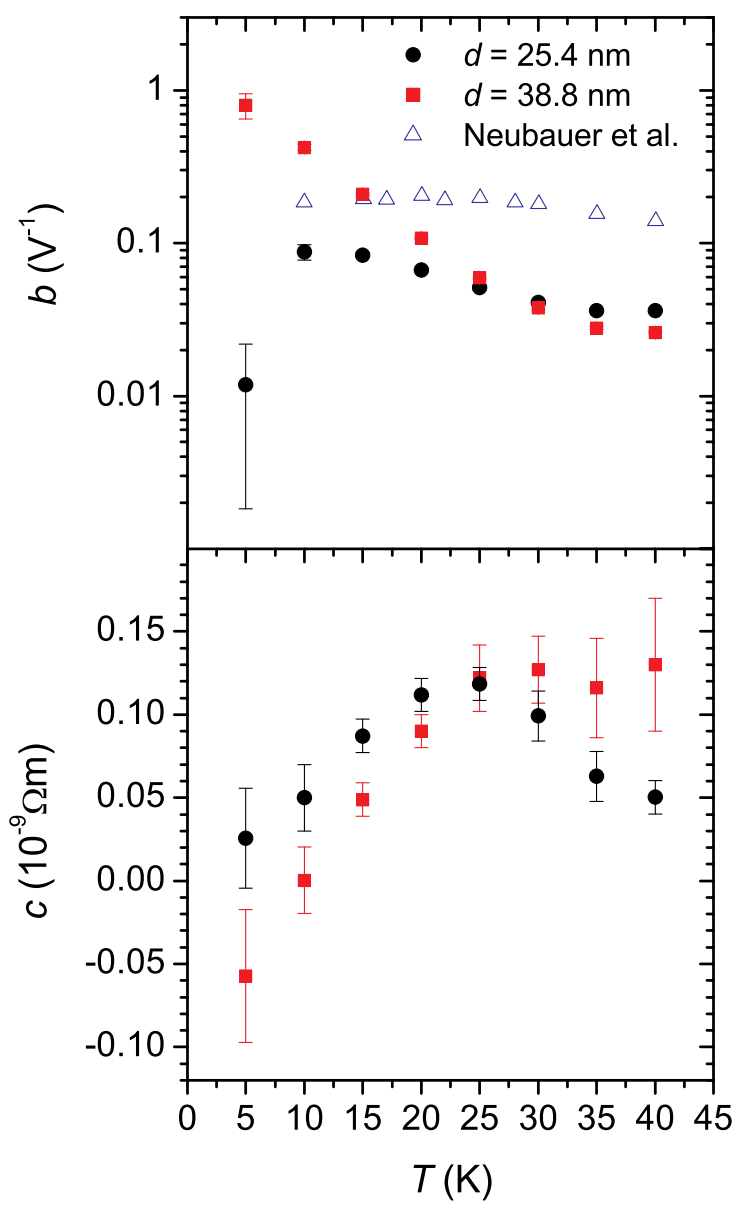

FIG. 7. (Color online) The solid points represent the parameters used to fit the anomalous Hall effect data as a function of temperature for the $25.4 \mathrm{~nm}$ and $38.8 \mathrm{~nm}$ thick MnSi films. The $b$ parameter is compared to the intrinsic contribution of the anomalous Hall effect found in bulk MnSi crystals reported in Ref. 53 (open triangles).

logical model to explain the Hall effect in $\mathrm{MnSi}$ is likely due to fact that it does not account for scattering from a conical spin structure. Recently Porter et al. have used a giant magnetoresistance model to explain the magnetoresistance in the conical phase of $\mathrm{FeGe} / \mathrm{Si}(111)$ epilayers ${ }^{33}$. If the conduction electrons are unable to adiabatically follow the conical spin texture, spin mixing of the spin channels will give rise to additional sources of scattering. This contribution to $\rho_{x x}$ is proportional to $M^{2}$, as shown in Fig. 4(b). Indeed, the magnetoresistance is found to have an $M^{2}$ dependence in the cone phase as found for $\mathrm{FeGe} / \mathrm{Si}(111)^{33}$. The atypical $M^{3}$ dependence in $\rho_{y x}^{\text {other }}$ is then naturally explained as skew scattering $\rho_{y x}^{\text {skew }} \propto \rho_{x x} M$ from the conical phase. This analysis suggests that $\rho_{y x}^{\text {other }}$ is not a topological contribution, but is correction to the model of the AHE that accounts for differences in scattering in ferromagnets and helical magnets.

\section{TRANSMISSION ELECTRON MICROSCOPY}

Here we introduce a method to simulate images of skyrmions taken with a transmission electron microscope to judge the conditions under which they could be observed and which will enable images of skyrmions to be analysed quantitatively to give absolute values of the flux density as was done recently for flux vortices in superconductors ${ }^{54}$.

The magnetic contrast observed in an out-of-focus electron micrograph is determined by changes in the component of the magnetic flux density normal to the electron beam projected through the sample thickness. In the case of the cone phase found in $\mathrm{MnSi} / \mathrm{Si}(111)$ epilayers in out-of-plane magnetic fields (see Fig. 1(c)), the projected magnetic flux is constant across a domain and therefore no magnetic contrast would be observed in an electron micrograph.

The first step in calculating the appearance of electron micrographs is to find the vector potential. By splitting the specimen into an infinite number of magnetic dipoles and summing the vector potentials from each, the vector potential $\mathbf{A}(\mathbf{r})$ can be related to the magnetization $\mathbf{M}(\mathbf{r})$ via:

$$
\mathbf{A}(\mathbf{r})=\frac{\mu_{0}}{4 \pi} \int \mathbf{M}(\mathbf{R}) \times\left(\frac{\mathbf{r}-\mathbf{R}}{|\mathbf{r}-\mathbf{R}|^{3}}\right) \mathrm{d}^{3} \mathbf{R}
$$

where $\mathbf{r}$ and $\mathbf{R}$ are position vectors and $\mu_{0}$ is the permeability of free space. As this is a convolution, it is treated most simply as a multiplication in Fourier space ${ }^{55}$ :

$$
\widetilde{\mathbf{A}}(\mathbf{k})=-\frac{i \mu_{0}}{2 \pi} \frac{\widetilde{\mathbf{M}}(\mathbf{k}) \times \mathbf{k}}{k^{2}}
$$

where we use the convention that if $f(\mathbf{r})$ is a function in real-space, its Fourier transform is $\tilde{f}(\mathbf{k})=$ $\int f(\mathbf{r}) e^{-2 \pi i \mathbf{k} \cdot \mathbf{r}} \mathrm{d}^{3} \mathbf{r}$.

If Cartesian coordinates are introduced with $z$ parallel to the electron beam and $x, y$ in the plane of the specimen, the magnetic contribution to the phase shift the electron beam has suffered as it exits the specimen is related to the vector potential via the Aharanov-Bohm formula ${ }^{56}$ :

$$
\phi(x, y)=-\frac{2 \pi e}{h} \int_{-\infty}^{\infty} A_{z}(x, y, z) \mathrm{d} z .
$$

In Fourier space, this becomes:

$$
\widetilde{\phi}\left(k_{x}, k_{y}\right)=-\frac{2 \pi e}{h} \widetilde{A}_{z}\left(k_{x}, k_{y}, 0\right) .
$$


and thus

$$
\widetilde{\phi}\left(k_{x}, k_{y}\right)=\frac{i e d \mu_{0}}{h} \frac{\left[\widetilde{\mathbf{M}}_{\perp}\left(k_{x}, k_{y}\right) \times \mathbf{k}_{\perp}\right]_{z}}{k_{\perp}^{2}},
$$

where $d$ is the film thickness, $\mathbf{k}_{\perp} \equiv\left(k_{x}, k_{y}, 0\right)$ and $\widetilde{\mathbf{M}}_{\perp}\left(k_{x}, k_{y}\right)$ is the two-dimensional Fourier transform of the magnetization averaged along $z$ through the thickness of the sample.

Any expression for the magnetization may be used in the above equation but instructive analytical expressions for the image intensity may be obtained by using the leading-order approximation for the magnetization given by Mühlbauer et al. ${ }^{7}$ :

$$
\begin{array}{r}
\mathbf{M}(\mathbf{r})=\frac{M_{0}}{3} \sum_{j=1}^{3}\left(\mathbf{n}_{j 1} \cos \left(2 \pi \mathbf{Q}_{j} \cdot \mathbf{r}+\alpha_{j}\right)\right. \\
\left.+\mathbf{n}_{j 2} \sin \left(2 \pi \mathbf{Q}_{j} \cdot \mathbf{r}+\alpha_{j}\right)\right)
\end{array}
$$

where $M_{0}$ is the saturation magnetization, $\mathbf{n}_{j 1}$ and $\mathbf{n}_{j 2}$ are unit vectors which are mutually perpendicular to $\mathbf{Q}_{j}$ and to one another and $\alpha_{j}$ are constants giving the relative phase of each helix. The so-called triple-Q state is realized for $\alpha_{1}=\alpha_{2}=\alpha_{3}=3 \pi / 2$ and produces a hexagonal lattice similar to the skyrmion lattice shown in Fig. 1(b).

In a conventional electron microscopy experiment, the specimen is thinned to produce an electron-transparent sheet approximately $50 \mathrm{~nm}$ thick with the wavevectors $\mathbf{Q}_{j}$ lying in the plane of the sheet and the electron beam impinging normal to its surface. The magnetization in Eqn. 14 produces a phase shift which oscillates as a function of position in real space with a maximum phase shift given by $\phi_{0}=\mu_{0} M_{0} e d /(h Q)$. Values for $M_{0}$ and $Q$ in several materials are given in Table $I$ and $\phi_{0}$ is $0.04 \mathrm{rad}$ for $50 \mathrm{~nm}$ thick $\mathrm{MnSi}$ and $0.09 \mathrm{rad}$ for $\mathrm{Fe}_{0.5} \mathrm{Co}_{0.5} \mathrm{Si}$ showing that these are in the weak phase limit, $\phi_{0} \ll 1 \mathrm{rad}$. Thus the magnetic contribution to the wavefunction of the electrons as they emerge from the specimen can be related to the phase shift via:

$$
\psi_{0}(x, y)=\exp [i \phi(x, y)] \approx 1+i \phi(x, y) .
$$

For $50 \mathrm{~nm}$ thick FeGe, $\phi_{0}=0.85 \mathrm{rad}$ and the appearance of the image should be calculated without approximating the exponential in the above equation.

An in-focus image is the squared modulus of the exitplane wavefunction and it is immediately clear that such an image will show no contrast as $I_{0}(x, y)=\left|\psi_{0}(x, y)\right|^{2}=$ 1. In order to visualise skyrmions (or any other magnetic object), out-of-focus images must be taken. An out-offocus image is equivalent to propagating the exit wavefunction through free-space by a distance $\Delta f$, known as the defocus. This can be done using the Fresnel-Kirchoff integral 57 but as this is a convolution, the relationship between the exit-plane wavefunction $\psi_{0}$ and the defocussed wavefunction $\psi_{\Delta f}$ is more conveniently expressed
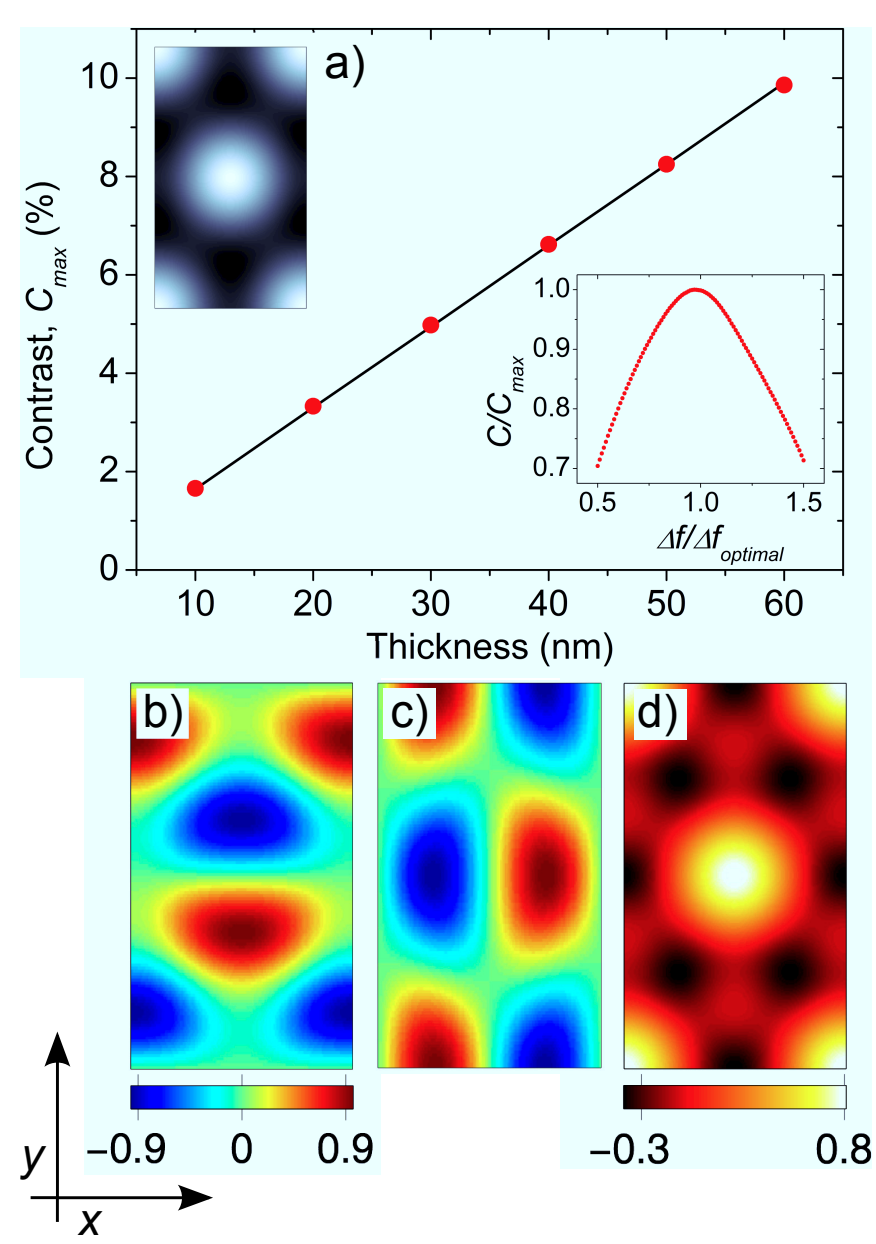

FIG. 8. (a) The maximum magnetic contrast, $C_{\max }$, from a skyrmion lattice produced in an electron micrograph under optimal defocus conditions for $300 \mathrm{kV}$ electrons. The red points are calculated from simulations of skyrmion lattices obtained from energy minimization calculations of a Dzyaloshinskii model of MnSi with the parameters given in Table I (see Ref. 11). The solid line is a fit to the calculations with Eq. (20). The right-most inset shows the dependence of the calculated contrast on the defocus setting. The leftmost inset shows one unit cell of the intensity distribution calculated from the simulations of the in-plane components of the magnetization averaged over the film thickness, (b) $M_{x}$, and (c) $M_{y}$ for a $d=10 \mathrm{~nm}$ film. The simulations are for a fixed magnetization modulus, in contrast to the triple- $\mathrm{Q}$ state whose variations in magnetization modulus $\Delta M / M$ are shown in $(d)$.

as a multiplication in Fourier space:

$$
\widetilde{\psi}_{\Delta f}\left(k_{x}, k_{y}\right)=\widetilde{\psi}_{0}\left(k_{x}, k_{y}\right) e^{-i \pi \lambda \Delta f k^{2}}
$$

where $\lambda$ is the electron wavelength. The intensity in the defocussed image is then $I_{\Delta f}(x, y)=\left|\psi_{\Delta f}(x, y)\right|^{2}$.

In the weak-phase approximation, Mühlbauer et al.'s model for the magnetization is simple enough to give an analytic solution for the intensity distribution in an out- 
of-focus image:

$$
\begin{aligned}
I_{\Delta f}(\mathbf{r})= & 1-2 \sin \left(\pi \lambda \Delta f Q^{2}\right) \frac{e d}{h Q} \frac{\mu_{0} M_{0}}{3} \times \\
& \sum_{j=1}^{3} \sin \left(2 \pi \mathbf{Q}_{j} \cdot \mathbf{r}+\alpha_{j}\right) .
\end{aligned}
$$

It can be seen that the contrast from the skyrmion array oscillates as a function of defocus and the defocus that gives the largest contrast is

$$
\Delta f_{\text {optimal }}=\frac{1}{2 \lambda Q^{2}} .
$$

At this defocus the contrast for the triple-Q states is

$$
C_{\max } \equiv \frac{I_{\max }-I_{\min }}{I_{\max }+I_{\min }}=\frac{3 e d}{2 h Q} \mu_{0} M_{0},
$$

Thus skyrmions are most readily observed in thick samples with a high magnetization and widely spaced skyrmions.

However, owing to the constructive and destructive interference of the helical waves in the triple- $Q$ construction, the ansatz contains spatial variations of the magnetization modulus, $\Delta M / M$, that span -0.3 to 0.8 (Fig. $8(\mathrm{~d})$ ), which cause a reduction in the magnetic image contrast. We therefore compare Eq. 19 to calculations of the contrast that use numerically calculated skyrmion lattices obtained from solutions to the Dzyaloshinskii model, the details of which are given in Ref. 11, together with Eqs. (13) - (16) and (18). These realistic simulations yield rigorous nonlinear solutions with fixed magnetization modulus (Fig. 8(b)and (c)). The calculations include twisting of the skyrmions near the surface that contribute to their stability. These twists do reduce the projected magnetization, and therefore also the contrast, although the reduction is small. The results of the simulations shown in Fig. 8(a) are found to be nearly linear in thickness and magnetization in the weak-phase limit, with optimal defocus settings, shown in the inset, that are the same as calculated in Eq. (18). Therefore we are able to correct the analytical results with a scaling factor $\gamma=1.58$,

$$
C_{\max }=\gamma \frac{3 e d}{2 h Q} \mu_{0} M_{0}
$$

We now compare several thin film materials listed in Table I in which skyrmions have been observed. The minimum detectable contrast is usually taken to be $3 \%{ }^{63}$ and we use this criterion to give a minimum thickness $d_{\min }$ below which skyrmions would likely be undetectable. We note that the thickness of the thin films measured for FeGe $(15 \mathrm{~nm}-75 \mathrm{~nm})^{17}, \mathrm{Fe}_{0.5} \mathrm{Co}_{0.5} \mathrm{Si}$ (several tens of $\mathrm{nm})^{16}$, and $\mathrm{MnSi}(\sim 50 \mathrm{~nm})^{18}$, are all above the minimum film thickness $d_{\min }$ where $C_{\max }=3 \%$ is expected. A comparison of the experimental images in Refs. 16-18 shows that, as predicted, images of skyrmions in $\mathrm{MnSi}$ are
TABLE I. The minimum thickness for the observation of skyrmions with optimal defocus settings for $300 \mathrm{kV}$ electrons calculated from Eq. 20

\begin{tabular}{lrrrrr}
\hline Material & Ref. & $\begin{array}{r}\mu_{0} M_{0} \\
(\mathrm{~T})\end{array}$ & $\begin{array}{r}1 / Q \\
(\mathrm{~nm})\end{array}$ & $\begin{array}{r}\Delta f_{\text {opt }} \\
(\mathrm{mm})\end{array}$ & $\begin{array}{r}d_{\text {min }} \\
(\mathrm{nm})\end{array}$ \\
\hline \hline FeGe & {$[58,59]$} & 1.0 & 70 & 1.24 & 0.8 \\
\hline $\mathrm{Fe}_{0.5} \mathrm{Co}_{0.5} \mathrm{Si}$ & {$[60,16]$} & 0.087 & 90 & 2.06 & 7.0 \\
\hline $\mathrm{MnSi}$ & {$[61,62]$} & 0.18 & 18 & 0.082 & 17 \\
\hline \hline
\end{tabular}

the noisiest, the noise is less for $\mathrm{Fe}_{0.5} \mathrm{Co}_{0.5} \mathrm{Si}$ and least for FeGe.

In the case of $\mathrm{MnSi} / \mathrm{Si}(111)$ thin films, $\mu_{0} M_{0}=$ $0.20 \mathrm{~T}^{14}$, and $1 / Q=14 \mathrm{~nm}^{30}$. For the $10 \mathrm{~nm}$ thick MnSi layer image in Fig. 1 of Ref. 28, the maximum contrast is $1.5 \%$, which is likely unobservable. If we use the wavelength $1 / Q=8.5 \mathrm{~nm}$ reported in Ref. 28 , we obtain $C_{\max }=0.9 \%$ making the observation of skyrmions even less likely. TEM micrographs of a $26.7 \mathrm{~nm}$ sample taken at room temperature are shown in Ref. 29, and reproduce all of the contrast features visible in Fig. 1 of Ref. 28. Given the temperature is far above $T_{C}=44 \mathrm{~K}$, these features are clearly not of magnetic origin, but rather are structural artifacts due to moiré fringes and surface defects. No new features could be discerned from the $d=26.7 \mathrm{~nm}$ sample in a defocus series taken at $T=10 \mathrm{~K}$. We note that the $C_{\max }=4 \%$ for this sample is just above the detection limit.

\section{CONCLUSION}

This Paper presents a consistent interpretation of the collection of measurements that exist for $\mathrm{MnSi} / \mathrm{Si}(111)$. One of the unresolved questions about MnSi epilayers was the origin of the anomalous Hall signal. We show that deviations of the Hall resistivity data from fits with the standard phenomenological model of the Hall effect, represented by a term $\rho_{y x}^{\text {other }}$, are not due to skyrmions. Our study finds a component of the Hall signal of the order $\rho_{y x}^{\text {other }} \simeq 5 \times 10^{-11} \Omega \mathrm{m}$ in the cone phase of $\mathrm{MnSi} / \mathrm{Si}(111)$ as well as bulk MnSi crystals. We interpret this contribution as an AHE due to skew scattering that results from nonadiabatic spin transport through a conical magnet. We hope that this work will motivate the development of a rigorous theoretical treatment of the anomalous Hall effect for conical magnets to account for differences in the scattering in the states above and below the saturation field.

Our analysis cautions about drawing conclusion on the existence of skyrmions from Hall effect measurements without consideration for the scattering from the conical texture. This raises questions about the existence of skyrmions in FeGe/Si(111) films in out-of-plane fields evidenced from Hall Effect measurement in Ref. 20. Con- 
trary to this original report of skyrmions in easy-axis films, Porter et al. find a hard-axis anisotropy for an $82 \mathrm{~nm}$ thick film, which would be expected to suppress skyrmions $^{33}$. Given the difficulty in extracting multiple contributions to $\rho_{y x}$ it is very difficult to prove the existence of skyrmions from Hall effect measurements alone. Supporting evidence from complementary techniques is important in the search for these solitonic states. To aid in this task, we provided a simple model for calculating the magnetic contrast in electron micrographs that can be used to guide the choice of material parameters in TEM experiments.

\section{ACKNOWLEDGMENTS}

We would like to thank Ulrich Rößler, Alex Bogdanov and Michael Robertson for many helpful discussions. T.L.M. and M.N.W. acknowledge support from NSERC and the support of the Canada Foundation for Innovation, the Atlantic Innovation Fund, and other part- ners which fund the Facilities for Materials Characterization, managed by the Institute for Research in Materials. Work done by J.C.L. was funded by the Royal Society. The work of F.N.R. was supported by RFBR, research project No. 14-02-31012.

\section{APPENDIX}

Here we provide measurements of the resistivity and magnetization that were used to fit the Hall effect data for the $25.4 \mathrm{~nm}$ thick MnSi sample in Sect. III. In Fig. 9 (a) we show the resistivity measured on cooling the sample from room temperature. The residual resistivity ratio of this samples is $\rho_{x x}(T=300 \mathrm{~K}) / \rho_{x x}(T=0 \mathrm{~K})=$ $183 \mu \Omega \mathrm{cm} / 6.65 \mu \Omega \mathrm{cm}=27.5$. The magnetoresistance is presented in Fig. 9 (b). The magnetic moment is obtained after subtraction of the substrate contribution as described $i^{12}$. The moment at zero field is determined by extrapolation of the high-field $M-H$ curves to zero field.
* j.c.loudon@gmail.com

$\dagger$ tmonches@dal.ca

1 A. N. Bogdanov and D. A. Yablonskii, Zh. Éksp. Teor. Fiz. 95, 178 (1989), [ Sov. Phys. JETP 68, 101 (1989) ].

2 A. Bogdanov and A. Hubert, J. Magn. Magn. Mater. 138, 255 (1994).

3 N. S. Kiselev, C. Bran, U. Wolff, L. Schultz, A. N. Bogdanov, O. Hellwig, V. Neu, and U. K. Rößler, Phys. Rev. B 81, 054409 (2010).

4 J. Sampaio, V. Cros, S. Rohart, A. Thiaville, and A. Fert, Nat Nano 8, 839 (2013).

5 N. Romming, C. Hanneken, M. Menzel, J. E. Bickel, B. Wolter, K. von Bergmann, A. Kubetzka, and R. Wiesendanger, Science 341, 636 (2013).

${ }^{6}$ X. Z. Yu, N. Kanazawa, W. Z. Zhang, T. Nagai, T. Hara, K. Kimoto, Y. Matsui, Y. Onose, and Y. Tokura, Nat Commun 3, 988 (2012).

7 S. Mühlbauer, B. Binz, F. Jonietz, C. Pfleiderer, A. Rosch, A. Neubauer, R. Georgii, and P. Böni, Science 323, 915 (2009).

8 H. Wilhelm, M. Baenitz, M. Schmidt, U. K. Rößler, A. A. Leonov, and A. N. Bogdanov, Phys. Rev. Lett. 107, 127203 (2011).

9 E. Moskvin, S. Grigoriev, V. Dyadkin, H. Eckerlebe, M. Baenitz, M. Schmidt, and H. Wilhelm, Phys. Rev. Lett. 110, 077207 (2013).

10 Y. Onose, Y. Okamura, S. Seki, S. Ishiwata, and Y. Tokura, Phys. Rev. Lett. 109, 037603 (2012).

11 F. N. Rybakov, A. B. Borisov, and A. N. Bogdanov, Phys. Rev. B 87, 094424 (2013).

12 S. A. Meynell, M. N. Wilson, H. Fritzsche, A. N. Bogdanov, and T. L. Monchesky, Phys. Rev. B 90, 014406 (2014).

13 A. B. Butenko, A. A. Leonov, U. K. Rößler, and A. N. Bogdanov, Phys. Rev. B 82, 052403 (2010).

14 E. Karhu, S. Kahwaji, T. L. Monchesky, C. Parsons, M. D. Robertson, and C. Maunders, Phys. Rev. B 82, 184417

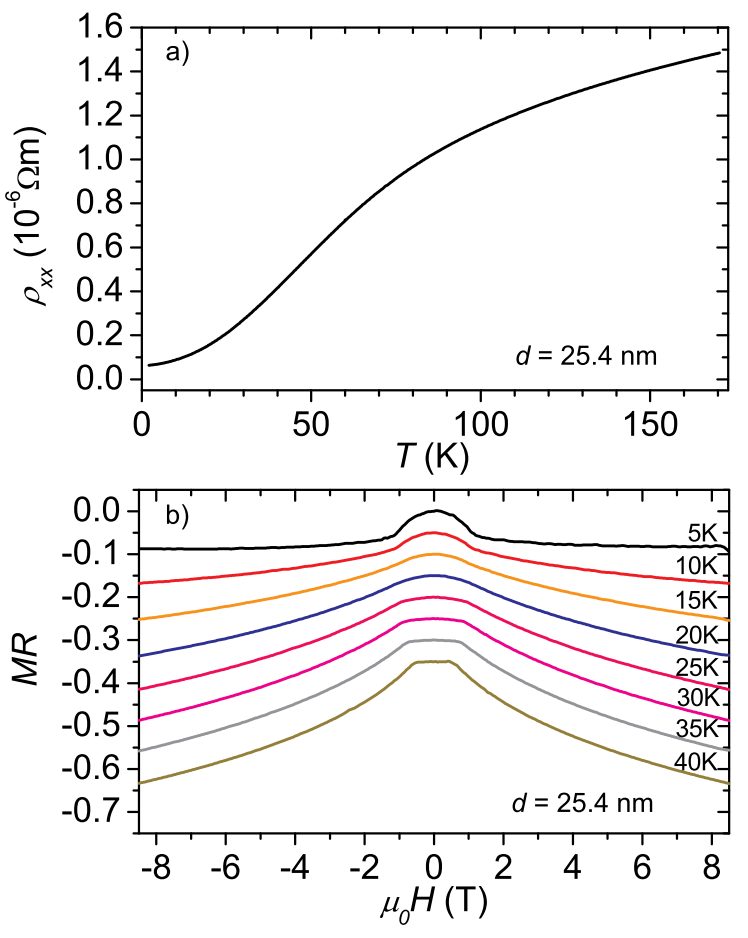

FIG. 9. (a) The temperature dependence of the longitudinal resistivity. (b) The magnetoresistance as a function of field for the $25.4 \mathrm{~nm}$ sample. Curves are offset by 0.05 each for clarity. The magnetoresistance is defined as $M R \equiv\left(\rho_{x x}(H)-\rho_{0}\right) / \rho_{0}$ and is a dimensionless quantity. 


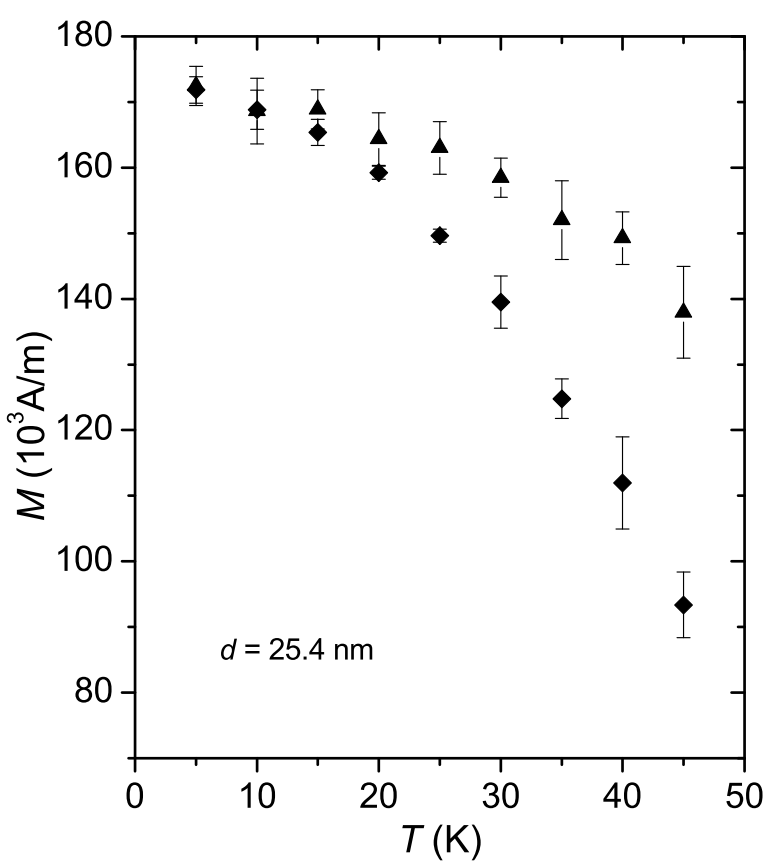

FIG. 10. The magnetization as a function of temperature for the $25.4 \mathrm{~nm}$ sample. The diamonds show the 0-field magnetization as found by extrapolating backwards from high-field $(2.5 \mathrm{~T}-4.5 \mathrm{~T})$. The triangles show the magnetization at $8.5 \mathrm{~T}$ found by extrapolating forwards in the $2.5 \mathrm{~T}$ to $4.5 \mathrm{~T}$ regime.

(2010).

15 M. N. Wilson, A. B. Butenko, A. N. Bogdanov, and T. L. Monchesky, Phys. Rev. B 89, 094411 (2014).

16 X. Z. Yu, Y. Onose, N. Kanazawa, J. H. Park, J. H. Han, Y. Matsui, N. Nagaosa, and Y. Tokura, Nature 465, 901 (2010).

17 X. Z. Yu, N. Kanazawa, Y. Onose, K. Kimoto, W. Z. Zhang, S. Ishiwata, Y. Matsui, and Y. Tokura, Nat. Mater. 10, 106 (2011).

18 A. Tonomura, X. Yu, K. Yanagisawa, T. Matsuda, Y. Onose, N. Kanazawa, H. S. Park, and Y. Tokura, Nano Lett. 12, 1673 (2012).

19 S. Heinze, K. von Bergmann, M. Menzel, J. Brede, A. Kubetzka, R. Wiesendanger, G. Bihlmayer, and S. Blügel, Nat. Phys. 7, 713 (2011).

20 S. X. Huang and C. L. Chien, Phys. Rev. Lett. 108, 267201 (2012).

21 M. N. Wilson, E. A. Karhu, A. S. Quigley, U. K. Rößler, A. B. Butenko, A. N. Bogdanov, M. D. Robertson, and T. L. Monchesky, Phys. Rev. B 86, 144420 (2012).

${ }^{22}$ H. Du, J. P. DeGrave, F. Xue, D. Liang, W. Ning, J. Yang, M. Tian, Y. Zhang, and S. Jin, Nano Lett. 14, 2026 (2014), http://pubs.acs.org/doi/pdf/10.1021/nl5001899.

23 G. E. Volovik, J. Phys. C: Solid State 20, L83 (1987).

24 J. Ye, Y. B. Kim, A. J. Millis, B. I. Shraiman, P. Majumdar, and Z. Tešanović, Phys. Rev. Lett. 83, 3737 (1999).

25 N. A. Porter, P. Sinha, M. B. Ward, A. N. Dobrynin, R. M. D. Brydson, T. R. Charlton, C. J. Kinane, M. D. Robertson, S. Langridge, and C. H. Marrows, ArXiv e- prints (2013), arXiv:1312.1722 [cond-mat.mes-hall].

26 C. Franz, F. Freimuth, A. Bauer, R. Ritz, C. Schnarr, C. Duvinage, T. Adams, S. Blügel, A. Rosch, Y. Mokrousov, and C. Pfleiderer, Phys. Rev. Lett. 112, 186601 (2014).

27 A. Neubauer, C. Pfleiderer, B. Binz, A. Rosch, R. Ritz, P. G. Niklowitz, and P. Böni, Phys. Rev. Lett. 102, 186602 (2009).

28 Y. Li, N. Kanazawa, X. Z. Yu, A. Tsukazaki, M. Kawasaki, M. Ichikawa, X. F. Jin, F. Kagawa, and Y. Tokura, Phys. Rev. Lett. 110, 117202 (2013).

29 T. L. Monchesky, J. C. Loudon, M. D. Robertson, and A. N. Bogdanov, Phys. Rev. Lett. 112, 059701 (2014).

30 E. A. Karhu, S. Kahwaji, M. D. Robertson, H. Fritzsche, B. J. Kirby, C. F. Majkrzak, and T. L. Monchesky, Phys. Rev. B 84, 060404 (2011).

31 M. N. Wilson, E. A. Karhu, D. P. Lake, A. S. Quigley, S. Meynell, A. N. Bogdanov, H. Fritzsche, U. K. Rößler, and T. L. Monchesky, Phys. Rev. B 88, 214420 (2013).

32 E. A. Karhu, U. K. Rößler, A. N. Bogdanov, S. Kahwaji, B. J. Kirby, H. Fritzsche, M. D. Robertson, C. F. Majkrzak, and T. L. Monchesky, Phys. Rev. B 85, 094429 (2012)

33 N. A. Porter, J. C. Gartside, and C. H. Marrows, Phys. Rev. B 90, 024403 (2014).

34 N. A. Porter, G. L. Creeth, and C. H. Marrows, Phys. Rev. B 86, 064423 (2012).

35 P. Sinha, N. A. Porter, and C. H. Marrows, Phys. Rev. B 89, 134426 (2014).

36 T. Yokouchi, N. Kanazawa, A. Tsukazaki, Y. Kozuka, M. Kawasaki, M. Ichikawa, F. Kagawa, and Y. Tokura, Phys. Rev. B 89, 064416 (2014).

37 J. Engelke, D. Menzel, and V. A. Dyadkin, J. Phys.: Condens. Matter 25, 472201 (2013).

38 M. D. Robertson, T. Burns, and T. Morrison, Micros. Soc. Can. Bull. 34, 19 (2006).

39 J. Smit, Physica 24, 39 (1958).

40 L. Berger, Phys. Rev. B 2, 4559 (1970).

41 Y. Tian, L. Ye, and X. Jin, Phys. Rev. Lett. 103, 087206 (2009).

42 N. Nagaosa, J. Sinova, S. Onoda, A. H. MacDonald, and N. P. Ong, Reviews of Modern Physics 82, 1539 (2010).

43 A. Crépieux and P. Bruno, Phys. Rev. B 64, 014416 (2001).

44 A. Shitade and N. Nagaosa, J. Phys. Soc. Jpn., J. Phys. Soc. Jpn. 81, 083704 (2012).

45 R. Karplus and J. M. Luttinger, Phys. Rev. 95, 1154 (1954).

46 M.-C. Chang and Q. Niu, Phys. Rev. B 53, 7010 (1996).

47 M. Onoda and N. Nagaosa, J. Phys. Soc. Jpn. 71, 19 (2002), http://dx.doi.org/10.1143/JPSJ.71.19.

48 M. Lee, Y. Onose, Y. Tokura, and N. P. Ong, Phys. Rev. B 75, 172403 (2007).

49 J. Weischenberg, F. Freimuth, J. Sinova, S. Blügel, and Y. Mokrousov, Phys. Rev. Lett. 107, 106601 (2011).

50 M. Lee, W. Kang, Y. Onose, Y. Tokura, and N. P. Ong, Phys. Rev. Lett. 102, 186601 (2009).

51 P. Milde, D. Köhler, J. Seidel, L. M. Eng, A. Bauer, A. Chacon, J. Kindervater, S. Mühlbauer, C. Pfleiderer, S. Buhrandt, C. Schütte, and A. Rosch, Science 340, 1076 (2013).

52 R. Ritz, M. Halder, C. Franz, A. Bauer, M. Wagner, R. Bamler, A. Rosch, and C. Pfleiderer, Phys. Rev. B 87, 134424 (2013). 
53 A. Neubauer, C. Pfleiderer, R. Ritz, P. Niklowitz, and P. Böni, Physica B 404, 3163 (2009), proceedings of the International Conference on Strongly Correlated Electron Systems.

54 J. C. Loudon, C. J. Bowell, N. D. Zhigadlo, J. Karpinski, and P. A. Midgley, Phys. Rev. B 87, 144515 (2013).

55 M. Beleggia and Y. Zhu, Phil. Mag. 83, 1045 (2003).

56 Y. Aharanov and D. Bohm, Phys. Rev. 115, 485 (1959).

${ }^{57}$ E. Hecht, Optics, 2nd edtn. (Addison-Wesley, Massachusetts, 1974).

58 L. Lundgren, K. Å. Blom, and O. Beckman, Phys. Lett. A 28, 175 (1968).
59 B. Lebech, J. Bernhard, and T. Freltoft, J. Phys.: Condens. Matter 1, 6105 (1989).

${ }^{60}$ Y. Onose, N. Takeshita, C. Terakura, H. Takagi, and Y. Tokura, Phys. Rev. B 72, 224431 (2005).

61 D. Bloch, J. Voiron, V. Jaccarino, and J. H. Wernick, Phys. Lett. A 51, 259 (1975).

62 Y. Ishikawa, K. Tajima, D. Bloch, and M. Roth, Solid State Commun. 19, 525 (1976).

63 M. Beleggia, Phys. Rev. B 69, 014518 (2004). 